\title{
Treatment Outcomes of Alternating Chemoradiotherapy for Nasopharyngeal Carcinoma
}

\author{
Hiromichi Gomi ${ }^{1}$, Mio Shinozaki ${ }^{1}$, Tatsuyuki Abe ${ }^{1}$, Yukinori Okada ${ }^{1}$, \\ Yasuo Nakajima ${ }^{1}$, Yoshihiro Akazawa ${ }^{2}$, Shigeru Kasugai ${ }^{2}$, and Masahiko Fukazawa ${ }^{2}$
}

(Received for Publication: March 2, 2015)

\begin{abstract}
Purpose: The aim of this study was to analyze the treatment outcomes of alternating chemoradiotherapy (ACRT) for nasopharyngeal carcinoma (NPC) and to assess the safety and efficacy of this treatment.

Materials and Methods: Twelve patients with stage II-IVB NPC were treated with ACRT in our hospital between 2005 and 2011. The radiotherapy period was divided into two components. In the first half, patients received 36 Gy to the whole neck field. In the second half, patients received 30 34.2Gy to the shrinking field to the primary and involved lymph nodes. A course of chemotherapy comprised of fluorouracil at a dose of 800 $\mathrm{mg} / \mathrm{m}^{2} /$ day was administered for 5 days (day 1-5), and cisplatin at $50 \mathrm{mg} / \mathrm{m}^{2} /$ day was administered for 2 days (day 6-7). A total of three courses of chemotherapy were performed systemically before starting radiotherapy, after completing the first half of radiotherapy and after completing the second half of radiotherapy.

Results: The planned radiation dose was achieved for all patients. Ten of the 12 patients completed the three courses of chemotherapy as planned. The remaining two patients completed only two courses because they refused, not due to adverse events. The five-year overall survival rate was $83 \%$, the progression-free survival rate was $67 \%$, the locoregional progression-free survival rate was $75 \%$, and the distant-metastasis free survival rate was $92 \%$. With regard to acute adverse events, the incidence of grade $\geqq 3$ hematotoxicity was $33 \%$, and the nonhematotoxicity event was only one case of grade 3 mucositis. No late adverse events of grade $\geqq 3$ occurred.

Conclusion: ACRT offered an excellent completion rate and could be safely conducted. Although a limited numbers of subjects were considered in this study, the outcomes of ACRT were favorable in terms of safety and efficacy.
\end{abstract}

\section{Key words}

Nasopharyngeal carcinoma, alternating chemoradiotherapy, radiotherapy

\section{Introduction}

Nasopharyngeal carcinomas (NPCs) are highly radiosensitive. Moreover, NPC lesions are located where surgery is technically difficult because of the proximity to the skull base. Therefore, radiotherapy is the first-line curative treatment for patients in early to advanced stages. Since both lymph node metastasis and distant metastasis occur frequently, chemotherapy is used as a rule. In recent years, treatment outcomes for carcinomas of the head and neck, particularly those in an advanced stage, have been improved through the use of chemoradiotherapy. Among methods of combining radiotherapy and chemotherapy, concurrent chemoradiotherapy (CCRT) has proved superior to treatment with radiotherapy alone in randomized controlled trials (RCTs) and meta-analysis ${ }^{1}$, and alternating chemoradiotherapy (ACRT) has also been shown to be effective ${ }^{2}$. As described above, NPC is characterized by frequent dis-

1 Department of Radiology, St. Marianna University School of Medicine

2 Department of Otorhinolaryngology, St. Marianna University School of Medicine 
tant metastasis, and high-dose chemotherapy is desirable for suppressing metastasis. In line with this notion, a method composed of CCRT using cisplatin (CDDP) and subsequent adjuvant chemotherapy (Adj) using CCDP and fluorouracil (5-FU) (CCRT +Adj) have been developed. This method has been demonstrated to be significantly more effective than radiotherapy alone in RCTs, and is now practically the standard method of treatment ${ }^{3-5)}$.

In ACRT, sufficient dosing in both radiotherapy and chemotherapy is expected to be feasible while maintaining a low incidence of adverse events by dividing the radiotherapy period into two components, alternating radiotherapy with chemotherapy. This approach is considered suitable for NPC treatment, and is widely performed in $\operatorname{Japan}^{6-8)}$. Several studies have reported higher or at least similar results of ACRT compared with those of CCRT+Adj. In 2005, our hospital adopted the same ACRT regimen used in the study of Fuwa et al. ${ }^{6}$ for treating NPC cases of stage $\geqq$ II. In this study, we retrospectively analyzed the outcomes of this treatment and compared them with previously reported results of standard CCRT +Adj therapy to assess the efficacy and safety of ACRT.

\section{Materials and Methods}

\section{Patients}

Subjects were 12 patients with NPC who underwent ACRT at our hospital between 2005 and 2011. Squamous cell carcinoma was confirmed in all patients and all NPCs were in stage II-IVB B according to the 6th edition of the Union for International Cancer Control (UICC) staging system. These 12 patients were all cases with NPC in stage $\geqq$ II, consecutively detected at our hospital between 2005 and 2011. Patients were in good general condition (the Eastern Cooperative Oncology Group performance status of $0-2$ ), with good bone marrow, kidney, and liver functions. MRI and CT were performed on all patients for evaluation of the progression of the primary and neck lesions. Chest and abdominal CT were performed for evaluation of the presence or absence of distant metastasis.

\section{Treatment methods}

Fig. 1 shows the treatment schedule. Patients were treated with ACRT in which radiotherapy and chemotherapy were performed alternately. The radiotherapy period was divided into two halves, while the chemotherapy was conducted in three parts: one before the first half of radiotherapy, one after finishing the first half, and the last one after the second half of radiotherapy was completed.

\section{Radiotherapy}

Each half of radiotherapy began two to three days after the preceding chemotherapy course was completed. Radiotherapy involved irradiation with 610MVX beams using a linear accelerator. The irradiation field in the first half of radiotherapy was the whole neck irradiation field from the skull base to the supraclavicular fossa. Patients were irradiated with daily fraction of 1.8-2Gy and received 36Gy of radiation in the first half. The second half of radiotherapy began after the second course of chemotherapy. The patients received 30-34.2Gy to the shrinking field to the primary lesion and involved lymph nodes. The to-

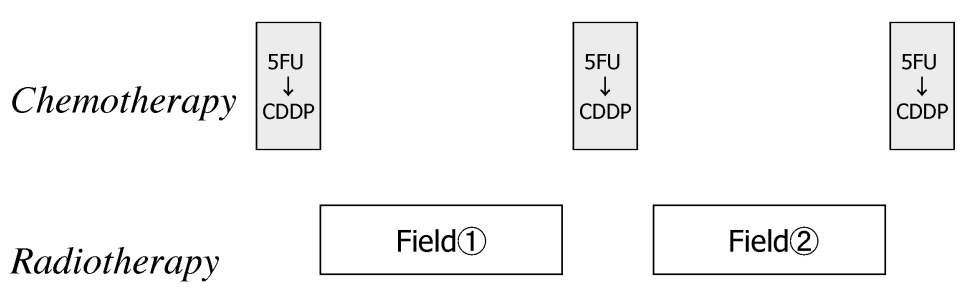

\begin{tabular}{llllllllllllll}
\hline Week & 0 & 1 & 2 & 3 & 4 & 5 & 6 & 7 & 8 & 9 & 10 & 11 & 12
\end{tabular}

Fig. 1. Treatment schedule of alternating chemoradiotherapy Chemothrapy: fluorouracil (5FU) $800 \mathrm{mg} / \mathrm{m}^{2}$ (day 1-5), cisplatin (CDDP) $50 \mathrm{mg} / \mathrm{m}^{2}$ (day 6-7) Radiotherapy: Field(1); whole neck field $1.8 \mathrm{~Gy} / \mathrm{f}$ total dose: $36 \mathrm{~Gy} / 20 \mathrm{f}$. Field(2); shrinking field to the primary lesion and involved lymph node 1.8 or $2 \mathrm{~Gy} / \mathrm{f}$ total dose: 30-34.2Gy. Field(1)+2) total dose: 66$70.2 \mathrm{~Gy}$ 
tal dose for the entire radiotherapy course was 66$70.2 \mathrm{~Gy}$.

\section{Chemotherapy}

In each course of chemotherapy, $800 \mathrm{mg} / \mathrm{m}^{2} /$ day of 5FU was administered for 5 days (day 1-5), and $50 \mathrm{mg} / \mathrm{m}^{2} /$ day of CDDP was administered for 2 days (day 6-7). The second and third courses began two to three days after the preceding radiotherapy was completed.

\section{Follow-up and analysis}

As a rule, follow-up observations were conducted at 1-month interval in the first year after treatment, at 2-month interval in the second and third years, and at an interval of four to six months from the fourth year onward. Follow-up imaging was conducted once a year, including neck and chest CT. Recurrence and distant metastasis were checked by MRI and positron emission tomography (PET)-CT as needed.

We assessed the completion rate for ACRT. As treatment outcomes, the locoregional progressionfree survival rate (LRPFS), overall survival rate (OAS), progression-free survival rate (PFS), and distant-metastasis free survival rate (DMFS) were computed with the Kaplan-Meier method. Recurrence patterns were also investigated. Adverse events were evaluated according to the National Cancer Institute Common Terminology Criteria for Adverse Events (CTCAE) v 3.0.

This study was conducted with approval by the Ethics Committee of St. Marianna University School of Medicine (approval no: 889).

\section{Results}

\section{Patient characteristics}

Table 1 shows the patient characteristics. The male to female ratio was 7:5. Pathological results indicated squamous cell carcinoma for all patients, and no type I case was found according to WHO classifications. The numbers of patients with T1, T2a, T2b, $\mathrm{T} 3$, and $\mathrm{T} 4$ were $5,1,1,0$, and 5 respectively. For $\mathrm{N}$ factor, $1,5,5$, and 1 patient were N0, N1, N2, and $\mathrm{N} 3$, respectively. With regards to stages, 1, 2, 3, 5, and 1 patients were stage IIa, IIb, III, IVa, and IVb, respectively. Performance status was evaluated to be $0 \sim 1$ in all patients. The median and range of age were 57 years and 41-77 years. The median and range of the observation period were 74 months and 41-105 months.
Table 1. Patient Characteristics

\begin{tabular}{|c|c|c|}
\hline \multicolumn{2}{|l|}{ Characteristics } & $n$ \\
\hline \multirow[t]{2}{*}{ Gender } & male & 7 \\
\hline & female & 5 \\
\hline \multirow[t]{3}{*}{ Performance status } & 0 & 10 \\
\hline & 1 & 2 \\
\hline & 2 & 0 \\
\hline \multirow[t]{3}{*}{ Histology* } & type I & 0 \\
\hline & II & 9 \\
\hline & III & 3 \\
\hline \multirow[t]{5}{*}{ T stage } & 1 & 5 \\
\hline & $2 a$ & 1 \\
\hline & $2 b$ & 1 \\
\hline & 3 & 0 \\
\hline & 4 & 5 \\
\hline \multirow[t]{4}{*}{$\mathrm{N}$ stage } & 0 & 1 \\
\hline & 1 & 5 \\
\hline & 2 & 5 \\
\hline & 3 & 1 \\
\hline \multirow[t]{5}{*}{ Stage } & II a & 1 \\
\hline & $\Pi$ b & 2 \\
\hline & III & 3 \\
\hline & $\mathrm{IVa}$ & 5 \\
\hline & $\mathrm{IVb}$ & 1 \\
\hline \multirow[t]{2}{*}{ Age } & & years \\
\hline & mean (range) & $57(41 \sim 71)$ \\
\hline
\end{tabular}

*According to the $\mathrm{WHO}$ criteria

\section{Feasibility}

The planned radiation dose was achieved for all patients. The total radiation dose ranged from 68 $70.2 \mathrm{~Gy}$, with a median value of $70.2 \mathrm{~Gy}$. Of all 12 patients, 10 patients completed the three courses of chemotherapy as planned, while two patients completed two courses only. Discontinuation of chemotherapy for the two patients was not due to adverse events, but because they refused to continue. One of the two patients refused the second course of chemotherapy, but did complete all radiotherapy and one course of chemotherapy after completing radiotherapy. The other patient refused the third course of chemotherapy after completing radiotherapy. The former was a stage II patient and experienced no recurrence or adverse event after 74 months post-treatment. The latter was a stage IVa patient, and developed lung metastasis 14 months after the treatment. Although the primary lesion and cervical region remained controlled, the patient died from the primary disease 41 months after ending treatment.

\section{Treatment outcomes}

Complete remission was achieved in all patients, but the disease relapsed in four patients. Recurrence 
occurred in the primary lesion in two patients, in the primary lesion and cervical region in one patient, and distant metastasis (lung) in one patient. The five-year LRPFS was $75 \%$ (95\% confidence interval, 50.599.7\%) (Fig. 2), and the five-year DMFS was 92\% (95\% CI, 77.6-100\%) (Fig. 3). In one of the two patients with recurrence in the primary lesion, the disease relapsed 36 months after the treatment, but was controlled by stereotactic radiotherapy with a cyber knife. This patient had no illness as of 91-months after treatment. The other patient had recurrence 7 months after the treatment, underwent systemic chemotherapy, and was surviving with cancer as of 57- months after treatment. In the patient with primary lesion and cervical region recurrence, the disease relapsed in the cervical region 8 months after the treatment and was controlled by neck dissection, but the patient had the primary lesion recurrence 24-months after treatment and died of the primary disease 38 months after treatment. In the case of distant metastasis, the patient developed lung metastasis 14 months after the treatment and died from the primary disease 41-month after treatment. A total of two patients thus far died as described above, the cause of death for both being the primary disease. The five-year OAS was $83.3 \%(95 \% \mathrm{CI}, 62.2-100 \%)$ and PFS was

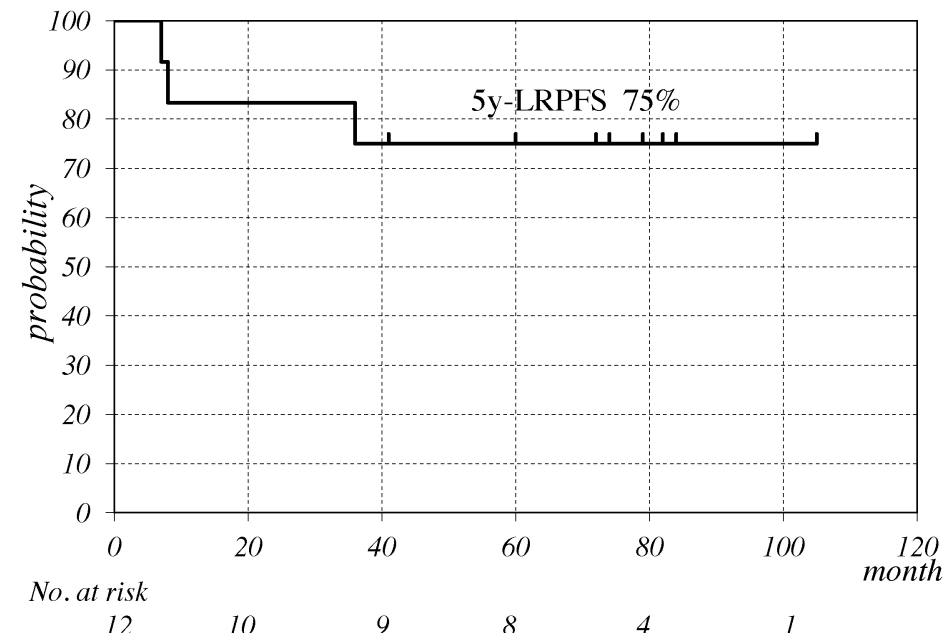

Fig. 2. Locoregional progression-free survival (LRPFS) rate

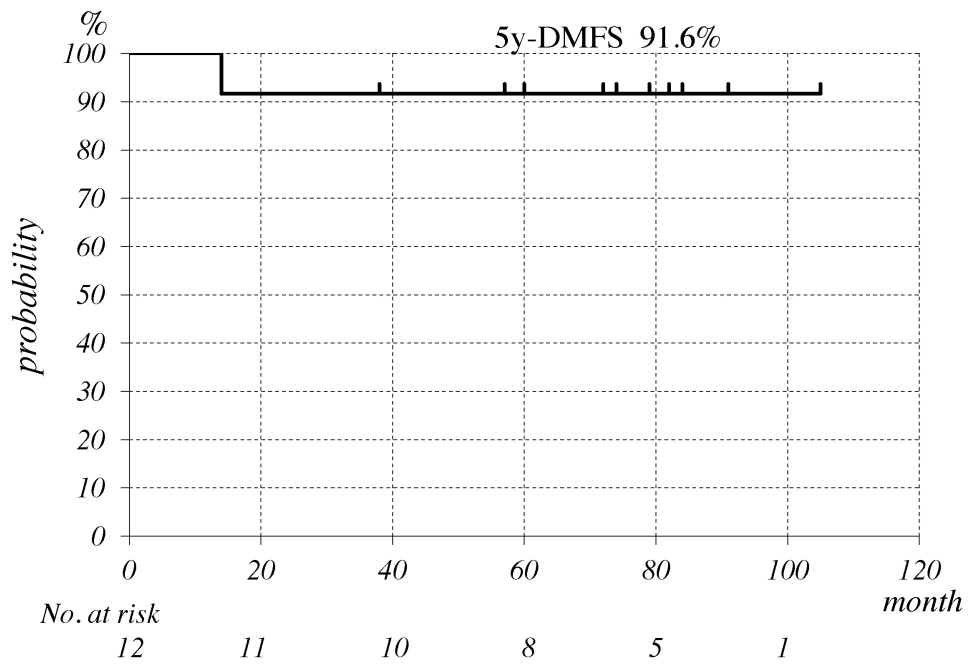

Fig. 3. Distant metastasis-free survival (DMFS) rate 
66.7\% (95\% CI, 40.4-93.4\%) (Fig. 4).

\section{Toxicity}

Table 2 shows adverse events. With regard to acute adverse events, hematotoxicity events included three cases of leukopenia and three cases of anemia of grade $\geqq 3$; the remaining cases were grade $\leqq 2$. With regard to non-hematologic adverse events, all patients experienced mucositis, dermatitis and dry mouth of grade $\geqq 1$, but the event of grade $\geqq 3$ was only one case of mucositis. All late adverse events were grade $\leqq 1$, except for two grade 2 cases of otitis middle and one grade 2 case of dry mouth. Dry mouth of grade $\geqq 1$ occurred in all patients as an acute adverse reaction. It improved over time, but persisted in many cases until the late stage.

\section{Discussion}

Radiotherapy is indicated for treatment of NPC at any stage because of its high susceptibility to radiation and associated anatomical conditions that make surgical treatment technically demanding. Yeh et al. reported treatment outcomes from radiotherapy alone in 849 patients divided according to the clinical stage $^{9)}$. According to their report, the five-year OAS was $82 \%$ for stage I, $72 \%$ for stage IIa, $71 \%$ for stage IIb, $55 \%$ for stage III, $42 \%$ for stage IVa and $39 \%$ for stage IVb. The five-year DFS was $76 \%$ for stage I, $72 \%$ for stage IIa, $65 \%$ for stage IIb, $47 \%$ for stage III, $31 \%$ for stage IVa and $30 \%$ for stage IVb, respec-

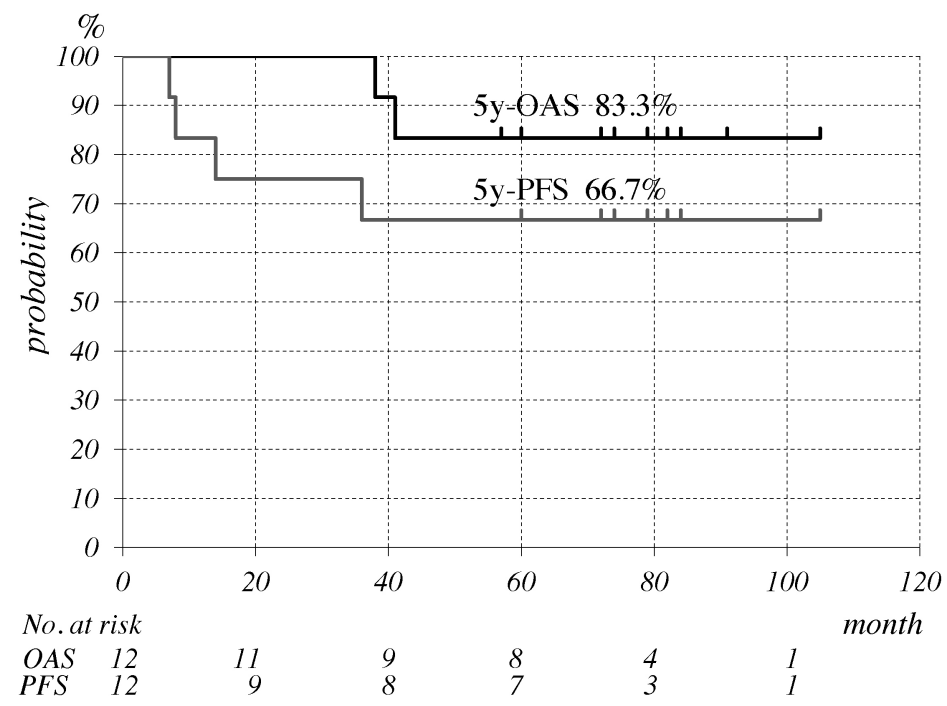

Fig. 4. Overall survival (OAS) and progression-free survival (PFS) rate

Table 2. Adverse Effects

\begin{tabular}{lllllll}
\hline Acute & Toxicity & 0 & 1 & 2 & 3 & 4 \\
\hline \multirow{4}{*}{ Leukopenia } & 1 & 4 & 3 & 4 & 0 \\
& Anemia & 5 & 1 & 3 & 3 & 0 \\
& Thrombocytopenia & 11 & 1 & 0 & 0 & 0 \\
& Mocositis & 0 & 8 & 3 & 1 & 0 \\
& Dermatitis & 0 & 7 & 5 & 0 & 0 \\
& Dry mouth & 0 & 10 & 2 & 0 & 0 \\
\hline \multirow{2}{*}{ Late } & & & & & & \\
& Dry mouth & 2 & 9 & 1 & 0 & 0 \\
& Otitis middle & 10 & 0 & 2 & 0 & 0 \\
\hline
\end{tabular}


tively. The results are not satisfactory for stage $\geqq I I$, particularly for stage III and IV. The reported fiveyear LRPFS was $78.4 \%$ and the five-year DMFS was $74.7 \%$, although these were values for all disease stages combined. There seems to be a room for improvement also in terms of locoregional control and suppression of distant metastasis. Thus the combined use of radiotherapy and chemotherapy has been attempted for a long time to improve therapeutic outcomes.

Two meta-analysis studies on the effects of chemoradiotherapy versus radiotherapy alone for NPC showed that the addition of chemotherapy contributed to the five-year OAS by $4-6 \%$ and to the five-year PFS by approximately $10 \%$ compared to those in radiotherapy alone, and that the concurrent use of radiotherapy and chemotherapy (CCRT) was the most effective type of combination therapy ${ }^{10) 11}$. In CCRT studies selected in these meta-analyses, a commonly used regimen involved adjuvant chemotherapy following CCRT (CCRT+Adj), which is currently the standard treatment for NPC. The intent of this method is to perform intensive chemotherapy as adjuvant therapy after CCRT to make the most use of high-dose chemotherapy for suppression of distant metastasis and avoid a completion rate drop likely to occur if intensified chemotherapy is performed concurrently with radiotherapy. The upper part of Table 3 shows the outcomes in trial arms in major RTC studies comparing CCRT+Adj and treatment with radiotherapy alone ${ }^{3-5) 12}$. Most of these studies followed the regimen used in inter group study (IGS) 00993). Radiotherapy was performed in the conventional fractionation with a total dose of $70 \mathrm{~Gy}$, and the chemotherapy component of CCRT was a three-course administration of $100 \mathrm{mg} / \mathrm{m}^{2} \mathrm{CDDP}$ at three-week interval. For post-CCRT adjuvant therapy, 80 $\mathrm{mg} / \mathrm{m}^{2} /$ day of CDDP (day 1 ) $+1000 \mathrm{mg} / \mathrm{m}^{2} /$ day of 5FU (day 1-4) were administered in one course, and three courses were performed at four-week interval. In these four studies, the reported outcomes for CCRT+Adj were significantly better than those for radiotherapy alone, except for the study of Lee et al. ${ }^{12)}$. Subjects in these studies were stage III-IVb patients. The reported 2-3-year outcomes include OAS of $70-80 \%$, DFS of $69-72 \%$, LRPFS of $92-98 \%$, and DMFS of $76-87 \%$. Grade $\geqq 3$ or acute adverse events, however, occurred at high incidence rates ranging from 60 to $83 \%$. While the completion rate for radiotherapy was satisfactory, only $55-70 \%$ of patients completed the intended number of chemotherapy courses, representing a problem remaining to be solved.

Here, we evaluated the suitability of the ACRT regimen adopted by our hospital for treating NPCs, in which sufficient doses are administered by dividing the radiotherapy period into two components, alternating radiotherapy with chemotherapy. Because no

Table 3. Treatment Outcomes of CCRT+Adj or ACRT for Nasopharyngeal Carcinoma

\begin{tabular}{|c|c|c|c|c|c|c|c|c|}
\hline & Author & year & no of pts & stage & OAS $\%$ & PFS $\%$ & LRPFS \% & DMFS $\%$ \\
\hline \multicolumn{9}{|c|}{$\overline{\mathrm{CCRT}+\mathrm{Adj}}$} \\
\hline & $\begin{array}{l}\text { Al-Sarraf } \\
\text { IGS } 0099\end{array}$ & 1998 & 78 & III $\sim$ IVb & 3у 78 & 3у 69 & - & - \\
\hline & $\begin{array}{l}\text { Lee (12) } \\
\text { NPC9901 }\end{array}$ & \multicolumn{6}{|c|}{ NPC9901 } & 3y 76 \\
\hline & Wee (4) & 2005 & 111 & $\mathrm{III} \sim \mathrm{IVb}$ & $3 y 80$ & $3 y 72$ & - & $3 y \quad 87$ \\
\hline & Chen & 2008 & 158 & $\mathrm{III} \sim \mathrm{IVb}$ & $2 y 90$ & 2 y 85 & $2 y 98$ & $2 y 87$ \\
\hline \multicolumn{9}{|l|}{$\overline{\mathrm{ACRT}}$} \\
\hline & Fuwa (6) & 2007 & 87 & $\mathrm{II} \sim \mathrm{IVb}$ & $5 y 83$ & $5 y 75$ & $5 y 87$ & $5 y 88$ \\
\hline & Goto (7) & 2013 & 82 & $\mathrm{I} \sim \mathrm{IVb}$ & $5 y 78$ & $5 y 68$ & $5 y 79$ & $5 y 88$ \\
\hline & Ueno (8) & 2014 & 27 & $\mathrm{II} \sim \mathrm{IVb}$ & $5 y 74$ & $5 y 71$ & - & - \\
\hline & Present study & 2015 & 12 & $\Pi \sim \mathrm{IVb}$ & $5 y 83$ & 5y 67 & 5y 75 & 5 у 92 \\
\hline
\end{tabular}

Abbreviations: ACRT = Alternating chemoradiotherapy: CCRT $+\mathrm{Adj}=\mathrm{Concurrent}$ chemoradiotherapy and adjuvant chemotherapy: DMFS=Distant metastasis-free Survival rate: LRPFS=locoregional progression-free survival rate: OAS=Overall survival rate: PFS=Progression-free survival rate 
RCT has been conducted to compare the outcomes of ACRT and radiotherapy alone in NPC patients only, a RCT including other types of head and neck cancer in addition to NPC is desirable. Merlano et al. have compared ACRT, in which $\mathrm{CDDP}+5 \mathrm{FU}$ and radiotherapy were repeated alternately, to radiotherapy alone in patients with unresectable, locally advanced head and neck cancer, and reported that ACRT produced significantly better results based on the fiveyear OAS of $24 \%$ vs. $10 \%$, PFS of $21 \%$ vs. $9 \%$, and LRPFS of $64 \%$ vs. $32 \%{ }^{2}$. Fuwa et al., who played a central role in ACRT research in Japan, have reported an excellent three-year OAS of $80 \%$ in their early study of stage III-IV patients ${ }^{13}$. Following this report, a majority of global studies on ACRT for NPC have been reported from Japan. The lower part of Table 3 shows the results of various recent studies, including ours. All included studies focused on stage II-IVb cases, except for that by Goto et al. ${ }^{7}$. The treatment schedules in all four studies were similar. The treatment outcomes of our study (the five-year OAS, 83\%; PFS, 67\%; LRPFS, 75\%; and DMFS, $92 \%$ ) were comparable to those of the other three studies. When comparing the outcomes of ACRT to those of CCRT+Adj, one should be aware that subjects were in different stages of disease and CCRT +Adj was applied in cases of stage $\geqq$ III, whereas ACRT was mostly applied in cases of stage $\geqq$ II. Nevertheless, approximately $80 \%$ of the cases that received ACRT were stage $\geqq$ III in all studies. In contrast, the evaluation time point differed in favor of CCRT+Adj, as its outcomes were measured 2-3 years after treatment whereas all ACRT outcomes were measured after 5 years. Given this difference, we can conclude that the outcomes of both regimens were nearly equivalent. The five-year DMFS data of 88-92\% obtained using ACRT indicate its contribution to suppression of distant metastasis, which is an objective of high-dose chemotherapy.

Regarding acute toxicity, the incidence of hematotoxicity of grade $\geqq 3$ was $33 \%$ in our study. In addition, a case of mucositis (8\%) was the only non-hematotoxicity, demonstrating the safety of this treatment. In the other three ACRT studies, the incidence rates for hematotoxicity and non-hematotoxicity of grade $\geqq 3$ were $45-67 \%$ and $22-38 \%$, respectively. Meanwhile, the incidence rates for acute toxicity (both hematologic and non-hematologic) of grade $\geqq 3$ reported for CCRT+Adj were $63-83 \%$. Although late toxicity was described only in a limited number of reports, no late toxicity reactions of grade $\geqq 3$ occurred in our study. Moreover, Goto et al. have reported this rate to be only $4.8 \%$ using ACRT. For CCRT+Adj, however, Lee et al. have reported a $20 \%$ incidence of grade $\geqq 3$ cases, indicating that ACRT is associated with fewer adverse events.

With regard to treatment completion rates, both types of treatment demonstrated high completion rates for the radiotherapy component. However, the completion rates of the scheduled chemotherapy courses were $70-90 \%$ for ACRT and only $55-70 \%$ for CCRT+Adj, indicating that ACRT was superior in terms of the completion rate in the chemotherapy component.

Consequently, although limited numbers of subjects were considered in this study, the outcomes of ACRT were favorable in terms of safety and efficacy. In the future, a controlled clinical trial to compare the outcomes of ACRT with those of CCRT+Adj, the standard method of treatment, should be performed.

\section{References}

1) Pignon JP, Maitre A, Maikkard E, Bourhis J, on behalf of the MACH-NC collaborative group. Meta-analysis of chemotherapy in head and neck (MACH-NC): An update on 93 randomised trials and 17,346 patients. Radiat Oncol 2009; 92: 4-14.

2) Merlano M, Benasso M, Corvò R, Rosso R, Vitale $\mathrm{V}$, Blengio F, Numico G, Margarino G, Bonelli L, and Santi L. Five-year update of a randomized trial of alternating radiotherapy and chemotherapy compared with radiotherapy alone in treatment of unresectable squamous cell carcinoma of the head and neck. J Natl Cancer Inst 1996; 88: 583-589.

3) Al-Sarraf M, LeBlanc M, Giri P G, Fu K K, Cooper J, Vuong T, Forastiere AA, Adams G, Sakr WA, Schuller DE and Ensley JF. Chemoradiotherapy versus radiotherapy in patients with advanced nasopharyngeal cancer: phase III randomized Inter group study 0099. J Clin Oncol 1998; 16: 1310-1317.

4) Wee J, Tan E, Tai B, Wong H, Leong S, Tan T, Chua E, Yang E, Lee K, Fong K, Tan H, Lee K, Loong S, Sethi V, Chua J and Machin D. Randomized Trial of Radiotherapy Versus Concurrent Chemoradiotherapy Followed by Adjuvant Chemotherapy in Patients With American Joint Committee on Cancer/International Union Against Cancer Stage III and IV Nasopharyngeal Cancer of the Endemic Variety. J Clin Oncol 
2005; 20: 6730-6738.

5) Chen Y, Liu MZ, Liang SB, Zong JF, Mao YP, Tang LL, Guo Y, Lin A, Zeng X, and Ma J. Preliminary results of a prospective randomized trial comparing concurrent chemoradiotherapy plus adjuvant chemotherapy with radiotherapy alone in patients with locoregionally advanced nasopharyngeal carcinoma in endemic regions of china. Int J Radiat Oncol Biol Phys 2008; 71: 1356-1364.

6) Fuwa N, Shikama N, Hayashi N, Matsuzuka T, Toita T, Yuta A, Oonishi H, Kodaira T, Tachibana H, Nakamura T, and Daimon T. Treatment results of alternating chemotherapy for nasopharyngeal cancer using cisplatin and 5-fluorouracil-A phase II study. Oral Oncology 2007; 43: 948-955.

7) Goto Y, Kodaira T, Fuwa N, Mizogichi N, Nakahara R, Nomura M, Tomita N, and Tachibana H. Alternating chemoradiotherapy in patients with nasopharyngeal cancer: prognostic factors and proporsal for individualization of therapy. $\mathrm{J}$ Radiat Res 2013; 54: 98-107.

8) Ueno T, Endo K, Kondo S, Wakisaka N, Murono S, Ito M, and Yoshizaki T. Factors affecting outcomes of alternating chemoradiotherapy for nasopharyngeal cancer. Ann Otol Rhinol Laryngol 2014; 123: 509-516.

9) Yeh SA, Tang Y, Lui CC, Huang YJ, Huang EY. Treatment outcomes and late complications of 849 patients with nasopharyngeal treatment with radiotherapy alone. Int J Radiat Oncol Biol Phys
2005; 62: 672-679.

10) Langendijk J, Leemans C, Buter J, Berkhof J and Slotman B. The Additional Value of Chemotherapy to Radiotherapy in Locally Advanced Nasopharyngeal Carcinoma: A Meta-Analysis of the Published Literature. J Clin Oncol 2004; 24; 4604-4612.

11) Baujat B, Audry H, Bourhis J, Chan A, Onat H, Chua D, Kwong D, Al-Sarraf M, Chi K, Hareyama M, Leung S, Thephamongkhol K, Pignon J, MAC-NPC Collaborative Group. Chemotherapy in locally advanced nasopharyngeal carcinoma: An individual patient data meta-analysis of eight randomized trials and 1753 patients. Int J Radiat Oncol Biol Phys 2006; 64: 47-56.

12) Lee A, Lau W, Tung S, Chua D, Chappell D, Xu L, Siu L, Sze W, Leung J, Law S, Yau T, Au J, O'Sullivan B, Pang E, O S, Au G, and Lau J. Preliminary Results of a Randomized Study on Therapeutic Gain by Concurrent Chemotherapy for Regionally-Advanced Nasopharyngeal Carcinoma: NPC-9901 Trial by the Hong Kong Nasopharyngeal Cancer Study Group. J Clin Oncol 2005; 23: 6966-6975.

13) Fuwa N, Ito $Y$, Kodaira $T$, Matsumoto A, Kamat M, Furutani K, Tatibana H, Sasaoka M, and Morita K. Therapeutic results of alternating chemoradiotherapy for nasopharyngeal cancer using cisplatin and 5-fluorouracil: its usefulness and controversial points. Jpn J Clin Oncol 2001; 31: 589-595. 